\title{
A Proposed VUV Oscillator-based FEL Upgrade at Jefferson Lab
}

S. V. Benson, D. R. Douglas, P. Evtushenko, F. E. Hannon, C. Hernandez-Garcia, J. M. Klopf, R. A. Legg, G. R. Neil, M. D. Shinn, C. D. Tennant, S. Zhang and G.P. Williams

Jefferson Lab, 12000 Jefferson Avenue, Newport News, VA, 23606, USA

\author{
Abstract \\ Advances in superconducting linac technology offer the possibility of an upgrade of the \\ Jefferson Lab Free Electron Laser (JLab FEL) facility to an oscillator-based VUV-FEL that \\ would produce $6 \times 10^{12}$ coherent $100 \mathrm{eV}$ photons per pulse at multi-MHz repetition rates in \\ the fundamental. At present JLab operates a pair of oscillator-based continuous-wave Free \\ Electron Lasers (FELs) as a linac-based next generation light source in the IR and UV, \\ with sub-picosecond pulses up to $75 \mathrm{MHz}$. Harmonics upwards of $10 \mathrm{eV}$ are produced and \\ the fully coherent nature of the source results in peak and average brightness values that are \\ several orders of magnitude higher than storage rings. The accelerator uses an energy \\ recovered linac design for efficiency of operation. New style superconducting linac \\ cryomodules with higher gradient, combined with a new injector and beam transport \\ system allow the development of the FEL to higher photon energies. \\ Keywords free electron laser (FEL), energy recovered linac (ERL), ultrafast, \\ photoemission.
}

\section{Motivation \& Background}

The parameters of the machine proposed here are determined by the requirements of studies of ultrafast dynamics on the characteristic time-scales of electrons in nature [1]. Such studies require bright sources of light that can deliver GW peak powers on the femtosecond time-scale, with full transverse and longitudinal coherence. In the 10-100 eV range electronic excitation and dynamics can be studied, but source repetition rates of $\sim \mathrm{MHz}$ are required to build statistics in experiments which depend on photoemission (photon in - electron out) and X-ray emission spectroscopy (photon in - photon out), as their key tools. Some of the science involves studies of out-of-equilibrium dynamics, both linear and non-linear, which requires multiphoton excitations (pump - probe) which is also possible with high repetition rate FEL oscillators.

Due to the fundamental physics of materials, existing laser sources have insufficient photon energy to enable electrons to be excited across a high enough range of desired final 
energies, while synchrotron radiation sources are not bright enough to explore the photoexcited electrons with high enough resolution or on fast enough timescales.

$1 \mathrm{GW}$ for $10 \mathrm{fs}$ corresponds to 10 microJoules of energy, which at a photon energy of 100 $\mathrm{eV}$, is approximately $10^{12}$ photons. At $10 \mathrm{eV}$ it is $10^{13}$ photons. These can be provided by Free Electron Lasers and require peak currents in the few 100 ampere range [2], corresponding to bunch charges in the 10's of picoCoulomb range. Such electron beam parameters have already been achieved in state-of-the-art FELs at lower photon energies such as the one at Jefferson Lab.

We note, however, that in some, but not all applications, the optical bandwidth of the FEL is too large, and may require monochromators to stretch the optical pulse and narrow its bandwidth. 10 fs pulses, for example, have an intrinsic bandwidth (if Gaussian) of almost 200 $\mathrm{mV}$, which is 2 orders of magnitude higher than that required by photomission studies of high Tc superconductors.

\section{Facility Description}

The existing Jefferson Lab light source facility [3,4] upon which this upgrade is based, is shown schematically in Fig. 1. It comprises a DC photo-gun, booster, three linac modules, and two recirculation paths, each containing an oscillator-based FEL comprising an undulator at the center of a 32m optical cavity (round-trip frequency $4.678 \mathrm{MHz}$ ). The electron and photon beam parameters are given in Table 1, but we note that cryo-cooled mirrors are required to satisfy the higher repetition rates at full power per pulse. In the injector, which is shown at the top right of Fig. 1, electrons are created in bunches by photoemission from a cesiated GaAs wafer, and then accelerated to $10 \mathrm{MeV}$ in a booster. They then pass into a linac comprising three JLab cryomodules generating a total of $80-140 \mathrm{MeV}$ of energy gain. The first and third modules incorporate conventional five-cell CEBAF cavities, while the central module is based on new 
seven-cell JLab cavities [5]. The central module design has demonstrated $82 \mathrm{MV}$ of continuous acceleration, surpassing all previous such systems. In these modules, the electron beam is accelerated (energy recovered) off-crest (off-trough) so as to impose a phase-energy correlation on the longitudinal phase space. This is used in subsequent transport to longitudinally match the beam to the required phase space at the FEL (dump). That is to say, the bunch is kept relatively long during acceleration, compressed to high peak current just before the undulator, then temporally expanded before reinsertion into the energy recovery phase of the linac.

\begin{tabular}{|c|c|c|}
\hline & IR FEL & UV FEL \\
\hline $\begin{array}{c}\text { Photon energy range of } \\
\text { fundamental }\end{array}$ & $0.1-1.4 \mathrm{eV}$ & $1-3.4 \mathrm{eV}$ \\
\hline Photon energy per pulse & 100 microJoules & 20 microJoules \\
\hline Repetition rate & $4.678-74.85 \mathrm{MHz}$ & $4.678-74.85 \mathrm{MHz}$ \\
\hline Photon Pulse length & $100 \mathrm{fs}-2 \mathrm{ps}$ & \\
\hline FWHM) & & \\
\hline Nominal pulse bandwidth & & $80-2 \mathrm{ps}$ \\
\hline Electron Beam Energy & $80-140 \mathrm{MeV}$ & 60 picoCoulombs \\
\hline Charge per electron bunch & 135 picoCoulombs & \\
\hline
\end{tabular}

Table 1. Parameters of the JLab FEL

From the undulators inside the 32 meter long optical cavities, the electron bunches emit tunable narrow-band light, which is retro-reflected twice so that it can be propagated co-linearly with the electron bunches that follow, to produce gain by longitudinal modulation. At the downstream out-coupling mirror, $~ 10 \%$ of the fundamental optical power is extracted with full transverse, as well as longitudinal coherence [2]. This corresponds to a conversion of about $1 \%$ of the electron beam energy. The "spent” electron beam still carrying $99 \%$ of its energy is then re-circulated back into the linac for energy recovery before being dumped. 


\section{Potential upgrade path}

Increases in electron beam energy from continuing advances in superconducting technology result in increases in photon energy, which scales as electron beam energy squared until emittance limits are exceeded. With present designs, cryomodules similar to the ones used in the existing FEL can achieve $100 \mathrm{MV}$ of acceleration each [6]. Using a 2-pass recirculating linac scheme with new beam transport arcs around the ends, it is possible to conceive of a $600 \mathrm{MeV}$ electron beam, that, with appropriately scaled undulators, could make $100 \mathrm{eV}$ light in the fundamental. A schematic of such a system is shown in Fig. 2.

Upgrading the present FEL to a VUV-FEL requires the following:

(1) An injector and injection line that create and preserve a lower emittance beam.

(2) New higher gradient superconducting linac cryomodules.

(3) A recirculating electron-beam transport system with the ability to maintain beam brightness during the acceleration, transport and compression process by avoiding the impact of lattice aberrations (chromatic and geometric) and collective effects such as beam breakup (BBU), other wakefield/impedance effects (e.g. the microbunching instability (MBI), resistive wall, etc.), space charge, and coherent synchrotron radiation (CSR). This must be combined with a phase space management scenario which gives adequate bunch compression/peak current at the wiggler and appropriate provision for transverse matching.

(4) The FEL itself, comprising an optical cavity containing a suitable insertion device and with mirrors whose figure and finish are suitable for $100 \mathrm{eV}$ light.

The brightness achievable from such a system would be substantially in excess of that provided by $3^{\text {rd }}$ generation storage rings (Fig 3). 
It should be noted that success of this recirculation and energy recovery approach would have major implications in the cost of a $2.5 \mathrm{GeV} 4^{\text {th }}$ Generation Light Source (4GLS) by reducing substantially the amount of linac, RF, and cryogenics required. Thus a secondary goal of this program is to demonstrate important technology for future hard x-ray next generation User facilities. It requires only half the cryomodules, associated cryogenic refrigeration and associated tunnel. And the cost impact is significant even at the modest gradient described here: a seven-cell $1497 \mathrm{MHz}$ cavity at $20 \mathrm{MV} / \mathrm{m}$ accelerating two passes of $1 \mathrm{~mA}$ beam will draw 30 kW RF power without energy recovery, but (depending on the choice of the loaded cavity quality factor, $\mathrm{Q}_{\mathrm{L}}$ ) may draw only 1 to $2 \mathrm{~kW}$ with recovery. This represents a savings of $\sim 1 / 4 \mathrm{MW} \mathrm{RF}$ drive-a cost reduction of order 2.5 M\$-per cryomodule. Specifics for the overall benefits, if any, depend on many factors since the extra beam transport can have significant cost.

\section{The Injector}

The injector can be defined as the region of the accelerator where the beam is generated and where the electron energy is still low enough that space charge forces dominate the electron beam evolution. The parameters above specify an operating regime for the injector which balances space charge forces in the beam with the initial intrinsic or "thermal" emittance and a bunch length compatible with a downstream buncher and/or booster.

In order to realize the VUV-FEL performance presented here, the electron beam must have a bunch charge of $200 \mathrm{pC}$ and a normalized transverse emittance of less than $2 \mathrm{~mm}$ mrad. Operating at a $4.68 \mathrm{MHz}$ repetition rate this corresponds to a cw average beam current of around $1.0 \mathrm{~mA}$. The injector itself is made up of three components.

1) An electron gun, which produces and accelerates the initial electron bunch. The gun defines the intrinsic transverse emittance of the injector; this is the minimum normalized, 
transverse emittance achievable, 2) Emittance compensation which is designed to realign the phase space ellipses of the bunch after emission, minimizing the downstream projected transverse emittance, typically a solenoid, and 3) A buncher and /or a booster to compress and accelerate the bunch to the linac while maintaining the longitudinal and transverse beam quality required.

No electron gun technology has demonstrated the capability to deliver the combination of CW operation with milliamps of average current at the required beam quality and pulse format necessary for a VUV / Soft X-ray FEL. There are three potential gun technologies compatible with the VUV-FEL injector requirements for both beam quality and operational reliability. Each of these schemes assumes the use of a photocathode and a laser to generate the electron beam.

These are:

(1) A very high frequency (VHF) normal conducting (NC) RF gun injector, for example the LBNL 187MHz [7] and ELSA 144MHz [8] guns.

(2) A DC gun injector, as used at the JLab FEL [9], Cornell ERL [10] and in development for the KEK/JAEA [11] ERL light source.

(3) A superconducting radiofrequency (SRF) gun injector, for example the WiFEL [12], and Rossendorf guns [13], or Brookhaven SRF electron gun [14].

The complete injector will combine one of the above gun technologies with additional electron beam manipulation components to do emittance compensation, and a buncher and/or a booster prior to injection into the linac. These downstream components must not compromise the incoming beam quality. The key however, to the injector is the gun. One candidate for the electron gun is a new 500kV DC gun presently under construction at the JLab FEL, with improved geometry yielding elimination of insulator charging [15]. The design consists of two 
inverted ceramics which support a central cathode electrode. This layout allows for a load-lock system to be located behind the gun chamber. The electrostatic geometry of the gun has been designed to minimize surface electric field gradients and also to provide some transverse focusing to the electron beam during transit between the cathode and anode. The gun geometry was changed in such a way as to direct field emitted electrons (inevitable during HV conditioning), towards the metallic gun chamber wall and not towards the ceramic insulators, reducing the possibility of charge buildup. Furthermore, a ceramic with a lowered bulk resistivity will allow for charge bleed-off. Finally, new surface processing techniques developed at JLab for SRF applications will be used to reduce the amount of initial field emission from the electrodes themselves. Simulations of this gun with a booster containing several single-cell, low frequency cavities indicate that the 6D brightness can be improved by a factor of 10 over that achievable in the present JLab FEL injector. Furthermore, a booster design with multiple singlecells or few-cells is more flexible in terms of the electron beam parameters it can accept, resulting in a more universal configuration that can be used with any gun technology.

The beam dynamics of the DC gun have been more extensively modelled, and were the subject of the first genetic algorithm optimizations [16]. The results of these simulations showed that emittance compensation can be successfully utilized with DC photocathode guns, and this has been confirmed experimentally with the Cornell photo-injector, where measurements are in very good agreement with the numerical modeling [17]. It is important to note that to achieve the required small transverse emittance, emittance compensation and full shaping of the drive laser pulse will be required regardless of the kind of electron gun used. When emittance compensation is implemented correctly, the contribution of the cathode thermal emittance to the overall emittance becomes sizable. JLAB presently uses GaAs:Cs cathodes which have the 
smallest thermal emittance measured [18]. GaAs:Cs cathodes have not been successfully operated in any type of electron gun except DC photoguns, enhancing our interest in the inverted ceramic DC gun. Further improvements may be possible through the use of biased anodes may also be used to extend cathode life by preventing ions downstream of the gun propagating towards the cathode [19].

\section{High Gradient Linac.}

The electron beam energy that can be achieved from the linac is based on the available real estate for acceleration and the cavity gradients that can be achieved. It is the tremendous progress that has been achieved in SRF gradient performance that permits consideration of this program. When CEBAF accelerator construction started in 1990, each cryomodule containing eight accelerator cavities was specified at $20 \mathrm{MV}$ of acceleration. By the time the construction was completed the best modules were actually producing double this acceleration, $40 \mathrm{MV}$. Since that time substantial progress in the processing of cavities as well as better engineering designs permit $100 \mathrm{MV}$ acceleration per $10 \mathrm{~m}$ module. Such is the specification of the cryomodules for the CEBAF $12 \mathrm{GeV}$ Upgrade presently under construction [6]. In the existing FEL facility there is room for a three module linac so that $300 \mathrm{MeV}$ electron beams can be produced in a single pass. It may in fact be feasible to achieve energies higher than $300 \mathrm{MeV}$, and we will aggressively pursue the development of higher gradient operation as it will provide access to even shorter operating wavelengths than indicated in the baseline. The final design of the cryomodules will be determined based on our experience with $12 \mathrm{GeV}$ and also on the results of our BES-funded program to test linacs with a cell shape and damping better optimized for light sources. However, $300 \mathrm{MeV}$ is insufficient to reach the highest photon energies desired by many users so we provide for a second pass of the beam through the linac to double the energy. 


\section{Electron Beam Transport.}

The challenges for the electron beam transport mentioned in section 3, either have been or are being met in pulsed FEL systems and there is a consensus that they can be met in a continuous wave FEL driver with "linear" topology [20]. Preliminary analysis suggesting that the brightness objectives can be met in recirculation is presented in [21]. We briefly review results of this study, detail additional challenges, and present a notional approach for addressing the key issue of beam quality preservation during recirculation.

Use of recirculation introduces a number of challenges:

(1) The need for an appropriate (beam-quality preserving) injection merger.

(2) The potential impact of additional beam transport length; in particular, the effect of wakefields, environmental impedances (with their potential to aggravate multibunch instabilities (MBI)), and space charge.

(3) Additional complexity in longitudinal matching.

(4) Use of common transport for multiple beams (during energy recovery).

(5) Possible beam breakup (BBU) limitations.

(6) The impact of lattice aberrations, quantum excitation, and coherent synchrotron radiation (CSR).

Experience with operation of the IR/UV Upgrade FEL demonstrates that careful management of longitudinal phase space alleviates the impact of collective effects. For the VUVFEL we must transport and match the linac beam to (from) the arcs using a spreader (recombiner). Following separation of the various passes, we recirculate each beam using a $180^{\circ}$ bending arc comprised of several periods of FODO (quad-dipole-quad) cells. This circularizes the arc footprint for efficient space utilization, provides periodicity and symmetry for aberration management and tuning capability, and decompresses the bunch length so as to alleviate CSR. 
Details of the transport system are driven by the design and optimization process and informed by operational experience. Adequate performance is provided by using twelve dipolequad-dipole cells tuned to sixth-integer phase advance in the bending plane and quarter-integer phase advance in the non-bending plane. The arcs are then second order achromats, coupling error effects are suppressed (because of the split tunes), and the system momentum compactions can be transparently tuned using periodically spaced "subfamilies” of the quads. Sextupoles can be similarly used to adjust $\mathrm{T}_{566}$, eliminating the need for harmonic RF; analogous trims of other focusing elements serve to manage linear and nonlinear dispersion.

The use of periodic transport in a (vault-limited) $6 \mathrm{~m}$ radius ensures that the matched Twiss parameters and dispersions are small. This alleviates aberrations and error sensitivity, reduces response to CSR, and keeps the beam size small—even during recovery of a potentially large energy spread exhaust beam after the FEL. With fields of 10 kG, (corresponding to a $1 \mathrm{~m}$ radius bend at $300 \mathrm{MeV}$, and $2 \mathrm{~m}$ at $600 \mathrm{MeV}$ ) the dipoles are $1 / 4$ to $1 / 2 \mathrm{~m}$ long. These parameters are adequate to limit incoherent synchrotron radiation (ISR) effects to tolerable levels.

The return arc is identical to the first. Momentum compactions of both combine to debunch the beam without parasitic compression. The longitudinal phase space is thus stretched rather thinly, so care must be taken to control impedances; however, the peak current for the elongated bunch is very low, giving little driving term to couple to the environment. Additional analysis will be required to establish an impedance policy and certify that microbunching effects are avoided.

The backleg transport cleanly transports the beam to the return arc without introducing aberration effects. It also provides space for utility functions, such as diagnostic stations and path length control. In the full energy arc, the FEL will be embedded in the backleg as well. In 
studies to date, we have used a simple quadrupole FODO array to good effect; phase advance is chosen to assist in aberration suppression. Recombination and reinjection to the linac are performed in a manner similar to the original spreader.

Analysis of both chromatic and geometric aberrations across the full recirculation (linac to linac) indicates that they are adequately managed and that energy recovery can be successfully executed. Results of geometric aberration analysis indicate the transverse phase space remains regular well beyond the nominal emittance (to $100 \mathrm{~mm}$-mrad normalized) across a moderately large momentum range $( \pm 1 \%)$, so the core beam will remain undistorted, halo will propagate cleanly, and the system will show reasonable freedom from orbit dependences in the optics.

CSR-driven emittance dilution is conceptually avoided by chirping the bunch by accelerating on the rising portion of the waveform (and thereby also alleviating LSC effects), decompressing the chirped bunch during transport through arcs with $\mathrm{M}_{56}>0$, and providing isochronicity by recombining the beam with a staircase achromat with $\mathrm{M}_{56}<0$ just prior to reinjection. Effects of CSR in the $300 \mathrm{MeV}$ transport were simulated using ELEGANT [22]. The effects are modest at 200 pC, with little transverse effect and moderate but regular distortion in the longitudinal phase space. Though the effect of the wake is apparent longitudinally, it can be simply managed by adjusting the lattice higher order momentum compactions to compensate the CSR effects. This is done in analogy to the compensation of such effects in transverse phase space as proposed over a decade ago by Dowell [23], although—in as much as this transport is common to both the accelerated and recovered beams — care must be taken to insure this does not adversely influence the energy recovery process. The compensation can equally well be done at full energy in the final compression, where it will not influence energy recovery at all. The CSR 
tracking results indicate that CSR is adequately managed, and also indicate that lattice aberrations are not an issue.

While studies and design optimizations are still being pursued, it is clear that recirculation can be used successfully in the VUV-FEL design for significant cost reduction with little performance penalty. A successful demonstration will establish the basis for even greater benefits to a future hard x-ray next generation user facility.

\section{Oscillator-based Free Electron Laser}

The availability of a pulse repetition rate of $4.687 \mathrm{MHz}$ allows for the operation of the VUVFEL as an oscillator, with a cavity length of $\sim 32 \mathrm{~m}$. To overcome high losses a relatively long wiggler of 6 meter length is used. The wiggler wavelength of $2.5 \mathrm{~cm}$ is short enough that an in vacuo device is required and the polarization would be linear. These wiggler parameters enable wide tunability and relative insensitivity to changes in the energy spread and emittance over the energies of interest. The wiggler gap is also of sufficient width to mitigate resistive wall heating of the wiggler chamber. In-vacuum linearly polarized wigglers of the required wavelength and length have been built at Spring 8 in Japan [24]. A new type of wiggler being developed at Cornell University called the "DELTA” undulator [25] would allow operation with elliptical polarization, which would be quite useful for many material studies. If the $6 \mathrm{~m}$ length proves to be a challenge, several shorter wigglers can be built instead, with the appropriate matching magnets between them, as shown in Fig. 2.

In the analysis that follows we consider a particular wiggler and optical configuration, though we anticipate that additional designs will need to be modeled before determining a final design. The performance of this FEL was determined using a variety of codes to simulate the FEL interaction, two of which, based on 1-D approximations, have been extensively 
benchmarked against operating FELs. An additional code is a combination of Genesis 1.3 to model the FEL simulation in 3-D, and the Optical Propagation Code (OPC) to model the oscillator [26]. OPC provides the flexibility to look at the effects of mirror figure distortions, add intra-cavity apertures, and analyze a number of other features.

For the chosen wiggler, the FEL acts as a high-gain, low-Q oscillator, in that a small amount of power from the output is fed back to the start to seed the next pulse. The power is outcoupled through a hole in the center of the mirror downstream of the wiggler. This provides an advantage in tunability since for photon energies less than $12.4 \mathrm{eV}$, the mirrors are relatively broadband [27] and the wavelength is controlled by the beam energy and the wiggler parameters. At higher energies, the mirror coatings become narrow-band. The mirror substrates will be cryocooled silicon. To add tunability, we plan to use the multiple mirror design employed on the other FELs at JLab[28].

The wiggler and optical cavity parameters for the Genesis/OPC simulations at $12.4 \mathrm{~nm}$ (100 eV) are shown in Table 2.

\begin{tabular}{|l|l|}
\hline Wiggler period (cm) & 2.5 \\
\hline Number of periods & 240 \\
\hline Wiggler gap (cm) & 7 \\
\hline Emittance (microns) & 2 \\
\hline Energy spread (\%) & 0.15 \\
\hline Peak current (kA) & 1.44 \\
\hline Cavity length (m) & 32.04196 \\
\hline Mirror radii (cm) & 1.27 \\
\hline High reflector mirror radius of curvature (m) & flat \\
\hline Output coupler mirror radius of curvature (m) & 23.5 \\
\hline
\end{tabular}




\begin{tabular}{|l|l|}
\hline Hole radius (cm) & 0.03 \\
\hline Mirror reflectivity (\%) & 70 \\
\hline
\end{tabular}

Table 2 Wiggler and optical cavity parameters for $12.4 \mathrm{~nm}$ operation

3-D simulations with these parameters, assuming mirrors that absorb power but do not otherwise distort, indicate a lasing efficiency of $0.03 \%$ and an output energy per pulse of almost $40 \mu \mathrm{J}$. For comparison, the Advanced Light Source at Berkeley (into the same bandwidth) delivers of order $1.6 \mathrm{pJ}$ per 70 ps pulse, or $20 \mathrm{~mW}$ peak power, which is 11 orders of magnitude lower. At this wavelength, the saturated gain is roughly 3.5 per pass and the output saturates in 60 passes, as shown in Fig. 4. Actual performance will be poorer when the effects of mirror thermal distortion and vibration, as well as e beam slippage, are accounted for in the model; activities that are all planned in the near future. At $20 \mathrm{~nm}$, the performance is better, with the output energy increasing by about $5 \times$ and a saturated gain of 18.8. At this longer wavelength, the power fed back is only $3 \%$ of the output, so the mirror reflectivities, and even surface figure are less critical, nor does the cavity length have to be held to a particularly high tolerance. Output power limits normally set by thermal distortion in mirrors of continuous FEL oscillators are also mitigated by the small fraction of light that is recycled and the use of cryocooling. Nonetheless, the operation of an oscillator at these short wavelengths is challenging. Besides the aforementioned thermal distortion of the mirrors, which can be partially compensated by deforming them[28], there are technical challenges associated with the maintenance of surface figure and finish along the periphery of the hole in the outcoupler mirror. At $100 \mathrm{eV}$, the modeled heat density on the high reflector mirror is about $34 \mathrm{~W} / \mathrm{mm}^{2}$, below the limit that synchrotron Si optics are routinely exposed to[29], so laser damage on that mirror does not appear to be an issue, but damage along the outcoupler hole's periphery may be an issue at longer wavelengths, when the incident E-field is higher. As mentioned earlier, the high 
repetition rates naturally available from the linac will provide very high average brightness for the fundamental and especially for the high harmonics. The pulse length in the oscillator mode will be determined primarily by the electron bunch length, e.g. greater than $100 \mathrm{fs.} \mathrm{Our}$ measurements have shown that the bandwidth of the output will be close to transform limited. It should be noted that for even lower photon energies than modeled, where aluminum coatings can be employed, the absorption in the coatings decreases and the performance will improve. The calculated output is roughly an Airy pattern in appearance, with the number of lobes determined by the Fresnel number.

Optical beam transport in the range up to $30 \mathrm{eV}$ can be readily accommodated by normal incidence optics, and metal mirrors. Thus transporting the lower energy beams to user labs is straightforward. Above $30 \mathrm{eV}$, more grazing optics or multilayer optics are required, but the latter, having a narrow bandwidth, is less desirable for some applications.

\section{Summary}

We have described a new generation of light source that is enabled by upgrading an existing FEL, to produce photons of up to $100 \mathrm{eV}$ with a brightness that is many orders of magnitude higher than electron storage rings. This represents a game-changing tool for science. Due to the high repetition rate of $4.678 \mathrm{MHz}$, this machine will also have useful average power in the harmonics, which will extend the useful reach of the machine for research. Further, the implementation of this VUV-FEL enables critical R\&D on low emittance cw injectors, and recirculating electron beams, which has the potential both to lower costs, as well as improving performance of, next generation x-ray sources.

Acknowledgements

This work could not have been done without the support of the entire FEL team, and without discussions with many other colleagues at Jefferson Lab. Financial support was provided by the Office of Naval Research, the Army Night Vision Laboratory, the Air Force Research Laboratory, the Joint Technology 
Office, the Commonwealth of Virginia, and by the U.S. DOE Basic Energy Sciences under contract No. DE-AC05-060R23177. 


\section{References}

1. W. Eberhardt, W. and F. Himpsel, chairs of report: "Next Generation Photon Sources for Grand Challenges in Science and Energy”, Basic Energy Sciences Advisory Committee, U.S. Dept of Energy, May 2009. http://www.sc.doe.gov/bes/reports/files/NGPS_rpt.pdf

2. G. Neil et al. "Sustained Kilowatt Lasing in a Free-Electron Laser with Same-Cell Energy Recovery”, Phys. Rev. Letts 84, 662 (2000).

3. S.L. Hulbert and G. P. Williams, "Calculations of Synchrotron Radiation Emission in the Transverse Coherent Limit”, Rev. Sci. Instr. 80, 106103 (2009).

4. Stephen Benson, et al., "High Power Operation of the JLab IR FEL Driver Accelerator", Proc. PAC’07, Albuquerque, New Mexico, USA p. 79 (2007).

5. I. E. Campisi et al "Superconducting cavity development for the CEBAF upgrade", Proc. PAC’99, New York, USA p. 937 (1999).

6. C.E. Reece et al. "Optimization of the SRF cavity design for the CEBAF $12 \mathrm{GeV}$ upgrade”, Proc. SRF’07, Peking University, Bejing, China p. 536 (2007).

7. K. Baptiste et al., “A CW normal-conductive RF gun for free electron laser and energy recovery linac applications”, Nucl. Instrum. \& Methods A599, 9 (2009).

8. S. Joly et al., “A High-Brightness Photo-Injector for a Free Electron Laser proposal”, Proc. EPAC’88, Rome, Italy p. 257 (1988).

9. Carlos Hernandez-Garcia, et al., "Status of the JLab ERL FEL DC Photoemission Gun”, Proc. ERL’09, Ithaca, NY, USA p. 37 (2009).

10. M. Liepe, et al., "Latest Results and Test Plans from the $100 \mathrm{~mA}$ Cornell ERL Injector SCRF Cryomodule”, Proc. IPAC’10, Kyoto, Japan, (2010).

11. N. Nishimori, et al., "Development of a 500-kV Photo-cathode DC gun for ERL light sources” Proc. Linac 10 Tsukuba, Japan , paper THP111(2010).

12. R. Legg, et al., "SRF Photoinjector R\&D at University of Wisconsin", Proceedings of ERL09, p 45, Ithaca, New York, USA, (2010).

13. R. Xiang et al. "The ELBE Accelerator Facility Starts Operation with the Superconducting rf gun”, Proceedings of IPAC’10, Kyoto, Japan p. 1710 (2010).

14. E. Wang, et al. "Quantum Efficiency and Lifetime of GaAs cathode in SRF Electron Gun”, Proceedings of IPAC’10, Kyoto, Japan p. 1764 (2010)

15. Fay Hannon, et al., "An inverted ceramic DC electron gun for the Jefferson Lab FEL”, Proc. FEL’09, Liverpool, UK, p. 383 (2009).

16. I.V. Bazarov, C.K. Sinclair, "Multivariate optimization of a high brightness dc gun photoinjector", Phys. Rev. ST-AB 8, 034202 (2005).

17. I. Bazarov, et al., "Initial beam results from the Cornell high-current ERL injector prototype”, Proc. PAC’09, Vancouver, CA p. 683 (2009).

18. I.V. Bazarov et al., "Thermal emittance and response time measurements of negative electron affinity photocathodes", Journal of Applied Physics, 103054901 (2008)

19. J. Grames et al., "A biased anode to suppress ion back-bombardment in a DC high voltage photoelectron gun”, AIP Conference Proceedings 980110 (2009).

20. W. A. Barletta et al. "Free electron lasers: Present status and future challenges", NIMA A618 69 (2010).

21. Christopher Tennant and David Douglas, “JLAMP: Low energy recirculator v1.0”, Jefferson Lab Technical Note JLAB-TN-10-023 (2010).

22. M. Borland, "elegant: A Flexible SDDS-Compliant Code for Accelerator Simulation,” Advanced Photon Source LS-287, September 2000. 
23. David H. Dowell, “Compensation of bend-plane emittance growth in a 180 degree bend”, Proc. PAC’97, Vancouver, BC, Canada p. 1888 (1997).

24. T. Tanaka et al., “In-Vacuum Undulators”, Proc. FEL’05, Stanford, California, USA, p. 370 (2005).

25. A. Temnykh, “DELTA undulator magnet for Cornell Energy Recovery Linac”, Proc. PAC’09 Vancouver, BC, Canada, p. 324 (2009).

26. J. G. Karssenberg et al., “FEL-Oscillator Simulations with Genesis 1.3”, Proc. FEL’06 BESSY, Berlin, Germany, p. 407 (2006).

27. W. R. Hunter, "Reflectance Spectra of Single Materials”, in Vacuum Ultraviolet Spectroscopy 1, Vol. 31, Experimental Methods in the Physical Sciences, J.A. Sampson and D. L. Ederer, Eds. Academic Press, London (1998).

28. M. D. Shinn et al. "Design of the Jefferson Lab IR upgrade FEL optical cavity”, Nuclear Instruments and Methods A507 196 (2003).

29. Lin Zhang, Wah-Keat Lee, Michael Wulff and Laurent Eybert, "The performance of a cryogenically cooled monochromator for an in-vacuum undulator beamline”, J. Synchrotron Rad. 10313 (2003). 
Figure Captions.

Fig. 1. Layout of the JLab FEL facility, showing the injector at the top, the superconducting linac on the upper left, with 2 re-circulation paths each containing an oscillator-based FEL. Fig. 2. Schematic of the VUV-FEL, with the existing FEL shown in gray for scale. The 2 recirculation paths are shown offset for clarity. In the actual machine they would lie one above the other.

Fig. 3. Lightsource landscape for average spectral brightness, showing $2^{\text {nd }}, 3^{\text {rd }}$, and $4^{\text {th }}$ generation sources, with the JLab FEL operational parameters indicated. These generic plots were made using an electron beam energy of $3 \mathrm{GeV}$, a bending radius of $5 \mathrm{~m}$, and for $1 \mathrm{nC}$ electron bunches at $100 \mathrm{MHz}(100 \mathrm{~mA})$. Source sizes used were $500 \times 1000,10 \times 100$, and $5 \times$ 5 microns respectively for the 2,3 and $4^{\text {th }}$ generation sources. $3^{\text {rd }}$ generation sources were elevated by 500 for insertion devices, and $4^{\text {th }}$ generation sources were elevated by a further $10^{10}$ to account for longitudinal coherence.

Fig. 4. Gain curves at 12.4 and $20 \mathrm{~nm}$ shown as intracavity power as a function of the number of passes in the cavity. 


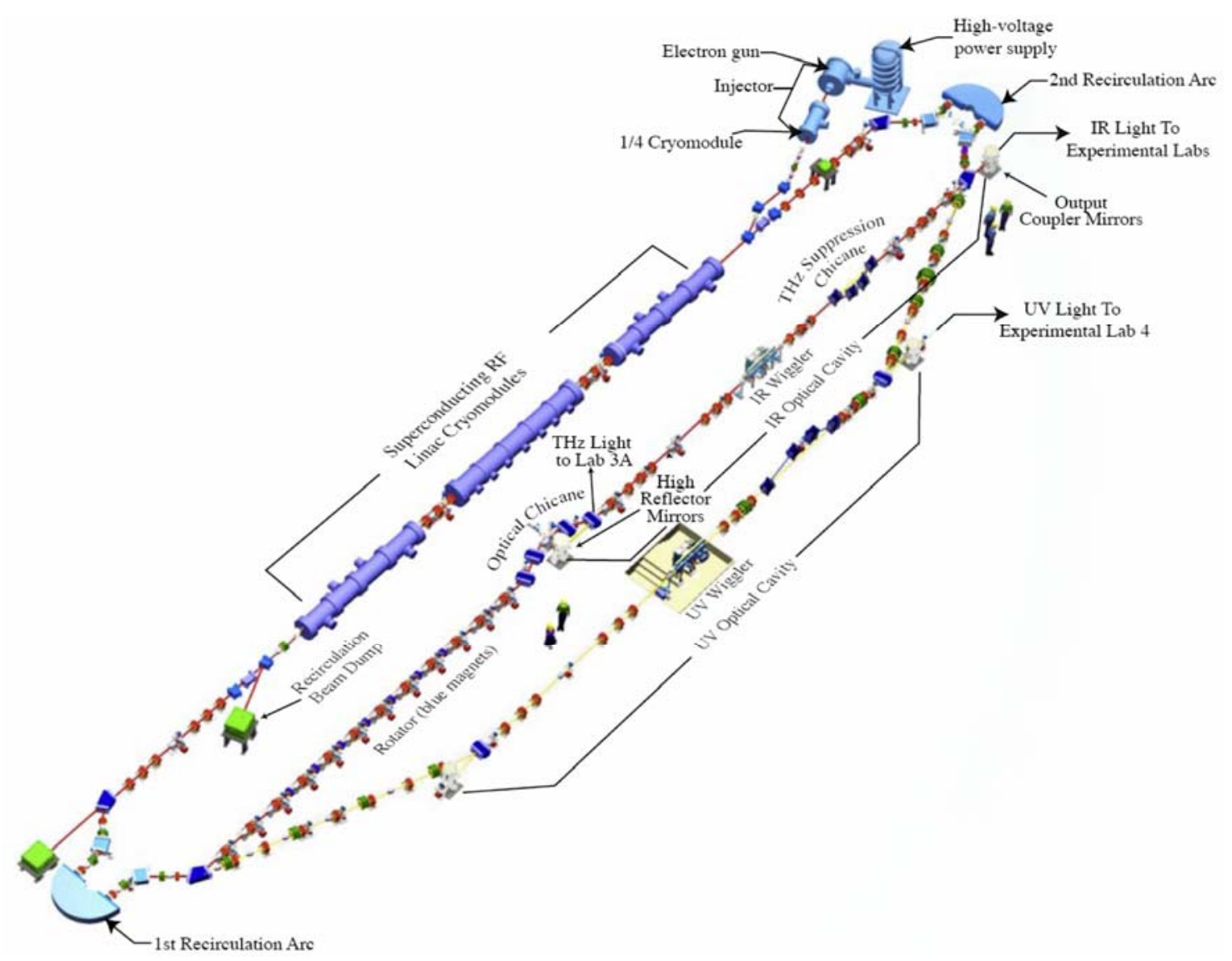

Benson et al. Fig. 1. 


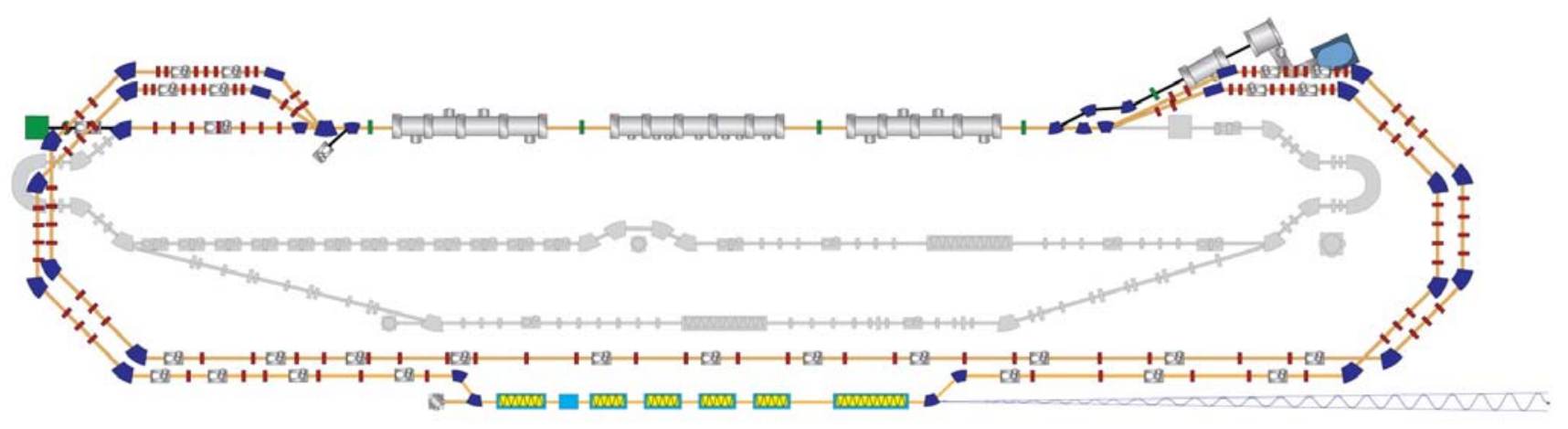

Benson et al. Fig. 2. 


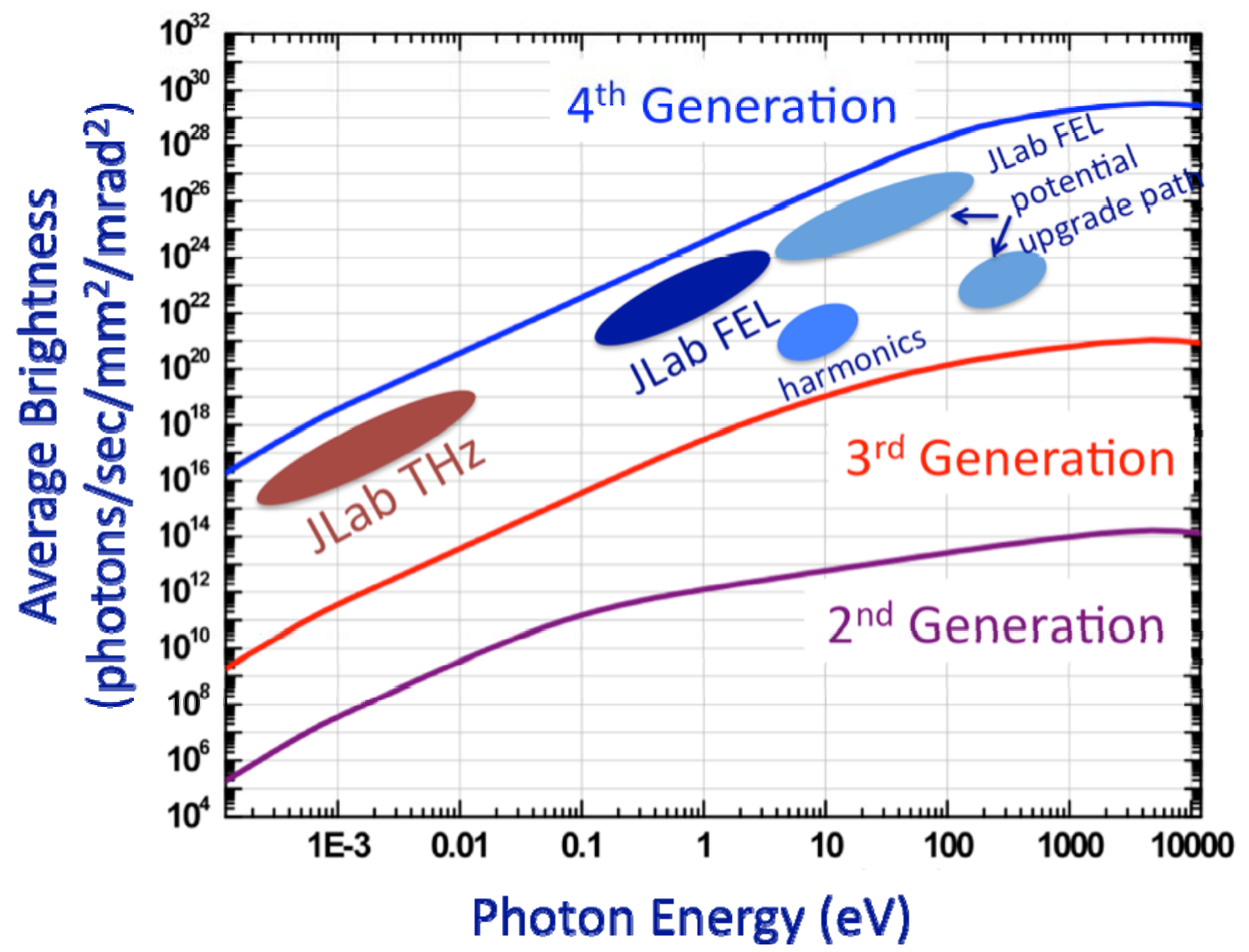

Benson et al. Fig. 3. 


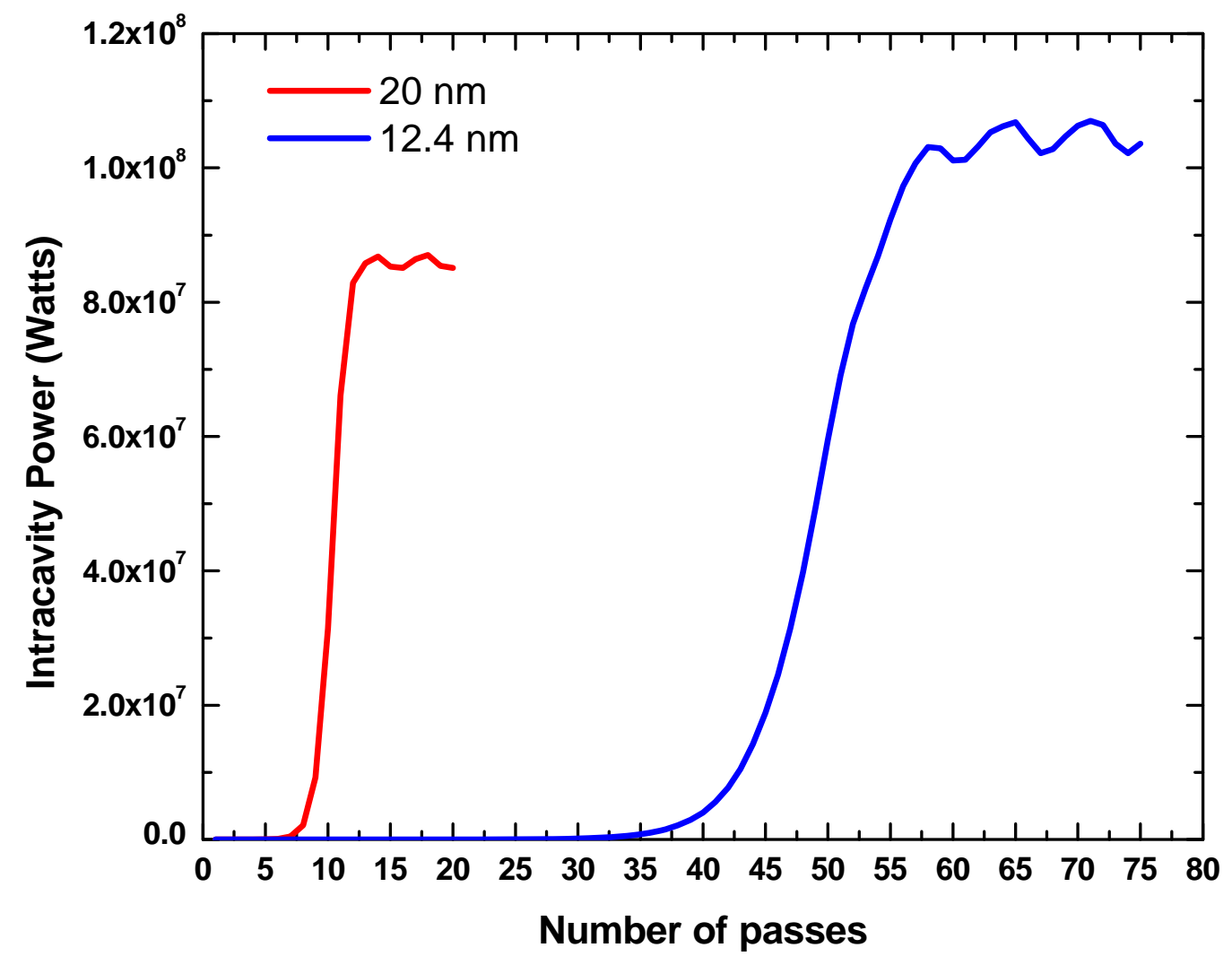

Benson et al. Fig. 4. 